\begin{tabular}{|l|l|}
\hline CS-6 & 1 \\
\hline
\end{tabular}

\title{
EXPERIMENTAL OSCILLATOR PHASE NOISE REDUCTION TECHNIQUE
}

\author{
H. Kimouche*
}

\begin{abstract}
This work presents a novel design methodology for reducing oscillator phase noise. This method is based on the choice of a topology of oscillator, as well as on the mastery of the nonlinear behavior of the active element. Obtained experimental results with the help of dielectric resonator oscillator (DRO) are presented for different kind of transistors.
\end{abstract}

\section{KEYWORDS}

Oscillators, Phase noise, Resonator, Cavities, Phase shifter.

*Teacher-Researcher, Dpt. Electronic, Microwaves/Radar Laboratory,
Polytechnic Military School, Algiers, Algeria.




\section{Introduction}

The growing development of microwave technologies, especially for markets of satellite telecommunications and communications between mobile, necessitates to design and realize microwave modules (Emission and Reception) being able to function to increasingly raised frequencies, with optimized performances in gain, in power and noise. So, demand of microwave sources with very high spectral purity becomes very important. Indeed, realization of microwave oscillators presenting a reduced phase noise remains one of the key points in the performance optimization research of current telecommunication systems.

To tempt to improve the spectral purity of an oscillator, the utilization of resonating structures with high quality factor in the feedback system is commonly admitted. From these oscillators topologies, several techniques exist that consist of adding particular circuits around the oscillator in a manner to reduce its phase noise. However, actually it does not exist a systematic method allowing to design an oscillator with a minimum phase noise from a given transistor and resonator. In this communication, we are going to expose a new method allowing to minimize the phase noise in dielectric resonator oscillators (DRO).

This method is based on the optimization of the nonlinear behavior of the active component, as well as on the choice of the appropriate oscillator topology.

This paper reports the first experimental results establish from a $10 \mathrm{GHz}$ oscillator.

\section{Phase noise}

Phase noise in oscillators is dominated by low frequency $1 / f$ noise up-conversion, which is caused by mixing effects between the carrier and the noise near DC which follows $1 / \mathrm{f}$ behavior, the common one being $1 / \mathrm{f}$.

Often, phase noise measurement specifications refer to single side band noise, denoted by a script $L\left(f_{m}\right)$, it is defined as being the ratio of the power of noise contained in a lateral band of $1 \mathrm{~Hz}$, situated at a distance $\mathrm{f}_{\mathrm{m}}$ from the carrier, and the power of the carrier, shown in figure (1): $\quad \mathrm{L}\left(\mathrm{f}_{\mathrm{m}}\right)=\left(\frac{P s s b}{P s}\right)_{1 H_{2}}$

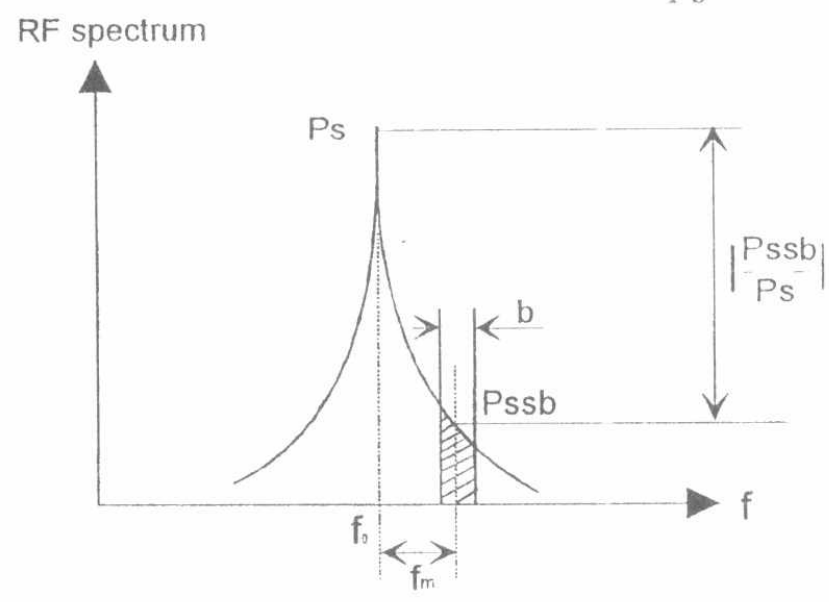

Fig.1. Spectrum of noisy oscillator

Where: $\quad$ Ps is the power of the carrier at fundamental.

Pssb is the level of power in the lateral band equal width $b$. 


\section{Principle of the method}

The principle of the method is to allow to define from a transistor and a given resonator, the topology of the free running oscillator, as well as large signal characteristics to privilege, so as to collect a minimum phase noise.

This principle applies indifferently to oscillators with bipolar or field-effect transistors. The resonator can be a metallic cavity or classic resonator. Obviously, the end result will depend directly on nonlinear characteristics and low frequency noise of the semiconductor component, as much as those of the resonator.

This method is based on two fundamental criteria :

$>$ The optimization of the transistor nonlinear behavior.

$>$ The choice of DR Oscillator topology.

Consider the topology of the oscillator, which the cavity functions in transmission. The diagram of an amplifier coupled to the cavity can be represented under the form shown in figure (2):

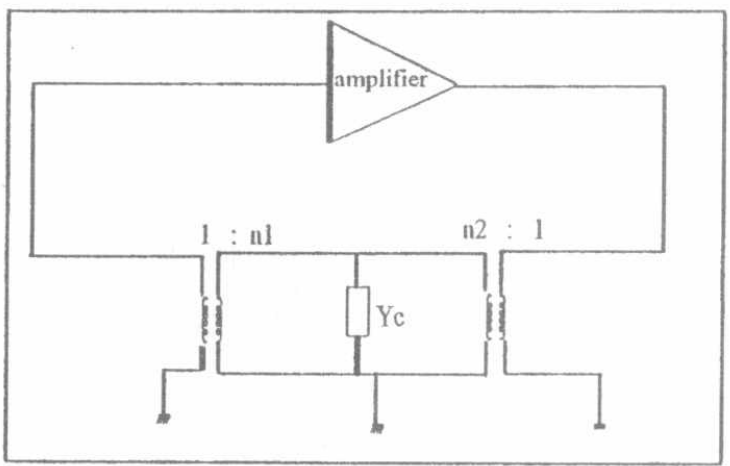

Fig.2. Basic circuit oscillator topology

The admittance $Y c$, the admittance of a parallel circuit $G_{r} L_{r} C_{r}$, represents the resonant circuit that is coupled to the amplifier with an ideal transformer, ratios $n_{1}$ and $\mathrm{n}_{2}$. The resonator factor $\mathrm{Q}_{\mathrm{r}}$ is then given by :

$Q_{r}=\left.\frac{\omega_{0}}{2 G_{r}} \frac{d B_{r}}{d \omega}\right|_{\infty}=\frac{\omega_{0} C_{r}}{G_{r}}$

Where $\left.\frac{d B_{r}}{d \omega}\right|_{\omega \infty}$ is the resonator slope factor.

The amplifier realized with a field-effect or bipolar transistor is adapted $50 \Omega$ in input and output for a large signal functioning. To realize the adaptation of the transistor, a simple solution consists of using ideal impedance transformers, that are going to return the impedance of $1 / G_{0}=50 \Omega$ at each access. In these conditions the amplifier can always be described at large signal analysis around the frequency of oscillation by the simplified diagram of the figure (3): 


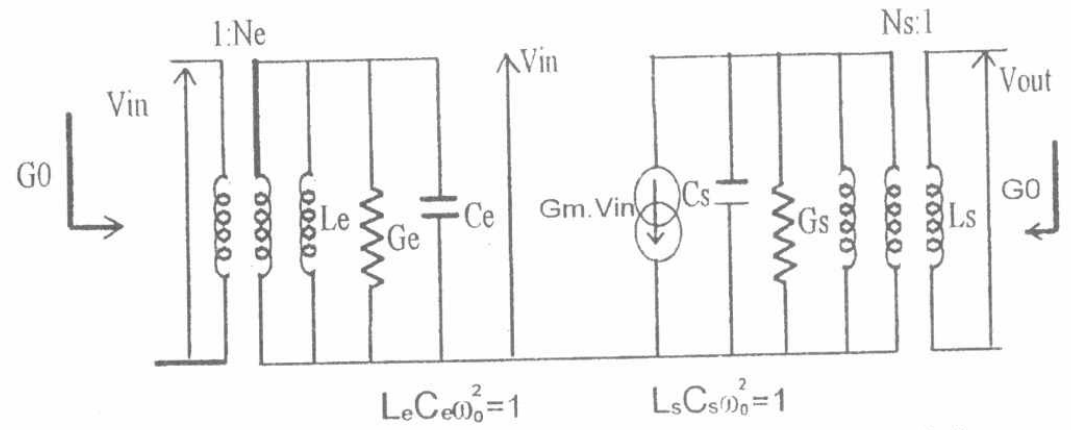

Fig. 3. Linear model of amplifier in oscillation state

However, as we move around the frequency $f_{0}$, we no longer maintain perfect adaptations in input and output of the amplifier. The elements introduced for the adaptation at $f_{0}$ return then an imaginary part :

$j \frac{d B}{d \omega} \Delta \omega$

with $\Delta \omega=\omega-\omega_{0}$

The diagram of figure (3) then becomes as shown in figure (4):

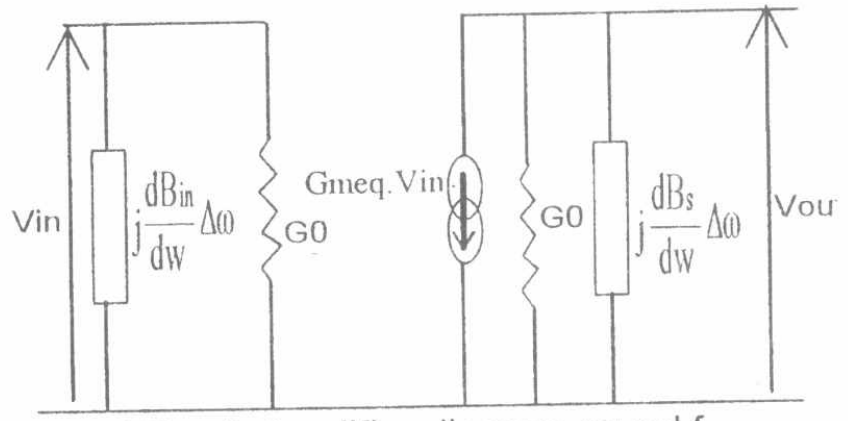

Fig. 4. Amplifier diagram round $f_{0}$

The oscillator diagram, described around the frequency $f_{0}$ of the system in autonomous regime, becomes then as shown in figure (5):

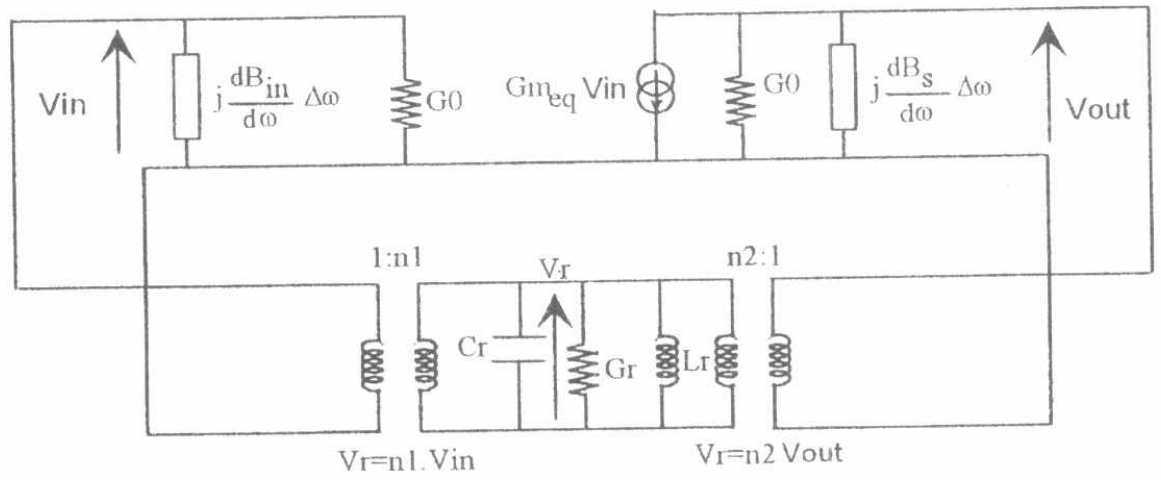

Fig.5. Oscillator diagram

The oscillation conditions at frequency $f_{0}$ are independent of the plane in which we define them. They can be calculated in any plane of the circuit and notably in the plane of entry of figure (5). Around $\omega_{0}$, we then obtains: 


$$
\begin{aligned}
& G_{0}+n_{1}^{2} \cdot G_{r}+\frac{n_{1}^{2}}{n_{2}^{2}} \cdot G_{0}-G m_{e q} \cdot \frac{n_{1}}{n_{2}}=0 \\
& {\left[\frac{d B_{i n}}{d \omega}+\frac{n_{1}^{2}}{n_{2}^{2}} \cdot \frac{d B_{s}}{d \omega}+n_{1}^{2} \cdot \frac{d B_{r}}{d \omega}\right] \Delta \omega=0}
\end{aligned}
$$

At $\omega_{0}$, the oscillation condition is reduced to:

$$
\mathrm{G}_{0}+\mathrm{n}_{1}^{2} \cdot \mathrm{G}_{\mathrm{r}}+\frac{\mathrm{n}_{1}^{2}}{\mathrm{n}_{2}{ }^{2}} \cdot \mathrm{G}_{0}-\mathrm{Gm}_{\mathrm{eq}} \cdot \frac{\mathrm{n}_{1}}{\mathrm{n}_{2}}=0
$$

Values of $n_{1}$ and $n_{2}$ have to be suitably chosen to optimize phase noise and are going therefore to depend from the point of the chosen characteristic $P_{\text {out }}=f\left(P_{\text {in }}\right)$ of the amplifier. The oscillator will be efficient in terms of phase noise that the dielectric resonator will be efficient. If we express the quality factor of the resonator alone according to stored energy and the power dissipated in the resonator, we can write :

$$
Q_{r}=2 \pi \frac{\text { stored energy }}{\text { dissipeted power by cycle }}=\omega_{0} \frac{\frac{1}{4} \frac{d B_{r}}{d \omega} V_{r}^{2}}{\frac{1}{2} G_{r} V_{r}^{2}}
$$

the oscillator efficiency $\eta$, with and without resonator, can be written then :

$$
\eta=\frac{\text { stored energy with resonator }}{\text { strored energy without resonitior }}=1+\frac{n_{1}^{2} \frac{d B r}{d \omega} \cdot V_{r}^{2}}{E_{\text {in }}+\frac{n_{1}^{2}}{n_{2}^{2}} E_{\text {out }}}
$$

Where $E_{\text {in }}$ is energy stocked at amplifier input.

$E_{\text {out }}$ is energy stocked at the amplifier output.

If we add an isolator at the amplifier end to maintain the load admittance constant, then the term $E_{\text {out }}$ does not intervene. The preceding relationship (6) simplifies and becomes:

$$
\eta=1+\frac{n_{1}^{2} \frac{d B_{r}}{d \omega} V_{r}^{2}}{E_{\text {in }}}
$$

We can from relationships (5) and (7) take conclusions : the resonator efficiency will be great that the term $\frac{d B_{r}}{d \omega}$ will be great. But for a given resonator (for a quality factor Qr perfectly definite), the fact to increase $\frac{d B_{r}}{d \omega}$ returns to maximize energy stored in the resonator. According to the definition of the quality factor $\mathrm{Qr}$, we have :

$\frac{1}{4} \frac{d B_{r}}{d \omega} V_{r}^{2}=\frac{1}{2} \frac{G_{r} Q_{r}}{\omega_{0}} V_{r}^{2}=\frac{Q_{r}}{\omega_{0}} \times$ dissipeted power in the resonator (8)

So the fact to store a maximum of energy returns also to have a maximal resonator dissipated power (at $Q_{r}$ constant).

The power dissipated in the resonator represents the difference between powers to transistor accesses. In other terms, the resonator power dissipated is equal to the maximal power added (Padd $\max$ ) of the transistor.

So, it is essential that transistor in oscillation state operates as close as possible at his maximal power added to obtain a minimum phase noise. 
In these conditions, the transistor diagrari of figure (5) can be considered as its diagram equal to the point of maximal pever added. In this point, Gmeq and Vin are related by:

$$
\frac{G m_{e q}{ }^{2} V_{i n}^{2}}{8 G_{0}}-\frac{1}{2} G_{0} V_{i n}^{2}=1 \text { cicld }_{\max }
$$

The input transistor voltage Vin is then fixed by the point at Padd $\max$ on the amplifier curve Pout $=\mathrm{f}(\mathrm{Pin})$ loaded by the isolator.

In conclusion to minimize the phase noise, two conditions are necessary :

- The active element has to function to provide its maximum power added.

- Energy stored in the resonator has to be transferred at the transistor input. This transfer is maximized by the adjustment of $n_{1}$ and $n_{2}$ values, as well as by the phase shift existing between access of the resonator and the transistor input.

\section{Experimental verifications}

We are going to verify these purposes with an experimental system developed with the topology where the cavity is in transmission as shown in figure (6):

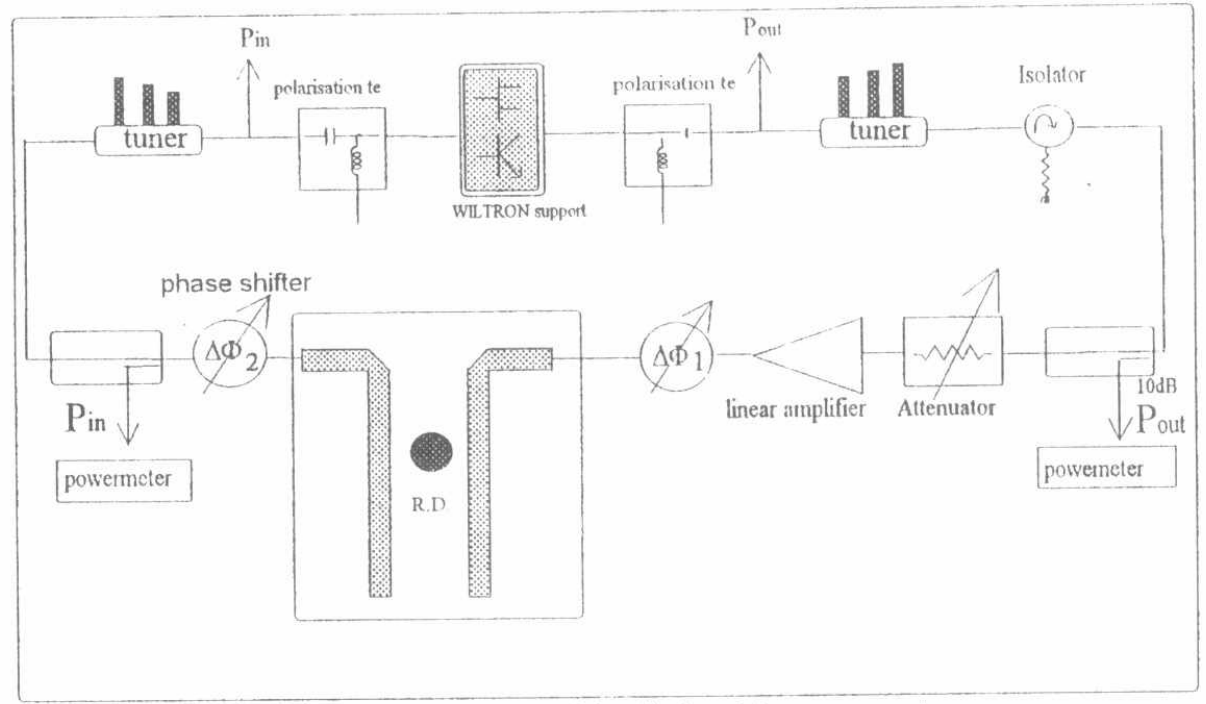

Fig.6. Oscillator with dielectric resonator

The characterization system is composed with the following elements :

- The cavity, composed with the dielectric resonator coupled to two microstrip lines, the resonance frequency is equal to $10 \mathrm{GHz}$.

- An amplifier with high gain, working in linear manner.

- Two tuners beforehand characterized.

- The transistor with his Wiltron support.

- Couplers, to collect and verify signals in input and output of transistor.

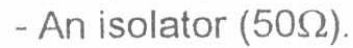

- Two phase shifter practically without losses, to control phase conditions feedback.

It is necessary to add the second phase shifter between cavity and amplifier in order to maximize the transfer of energy.

Phase noise was measured using the HP phase noise measurement system. We have used two kinds of transistor, a field effect transistor PHEMT (CENSA132) and a 
heterojunction bipolar transistor HBT (MJF4417), each transistor has been adapted in low level gain first, then in maximal power added.

A Bipolar transistor (Censa 132) has first been adapted in low-level gain. We have then measured the phase noise for a input power corresponds to the maximum power added. Then we have made vary this input power around that giving the maximum power added. The phase noise at $10 \mathrm{KHz}$ of the carrier is presented versus phase shift $\Phi_{2}$ brought by the phase shifter placed on the input transistor.

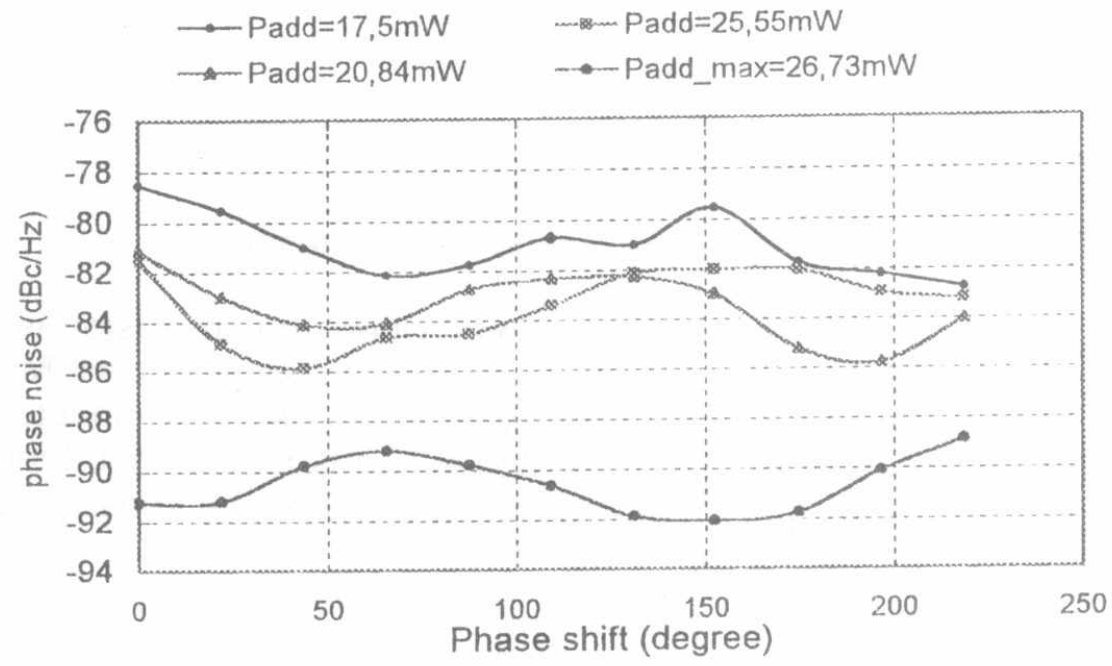

Fig. 7. Transistor adapted in low-level gain

We have proceeded the similar manner, but here the transistor is adapted in maximum power added. Obtained results are indicated on the figure (8):

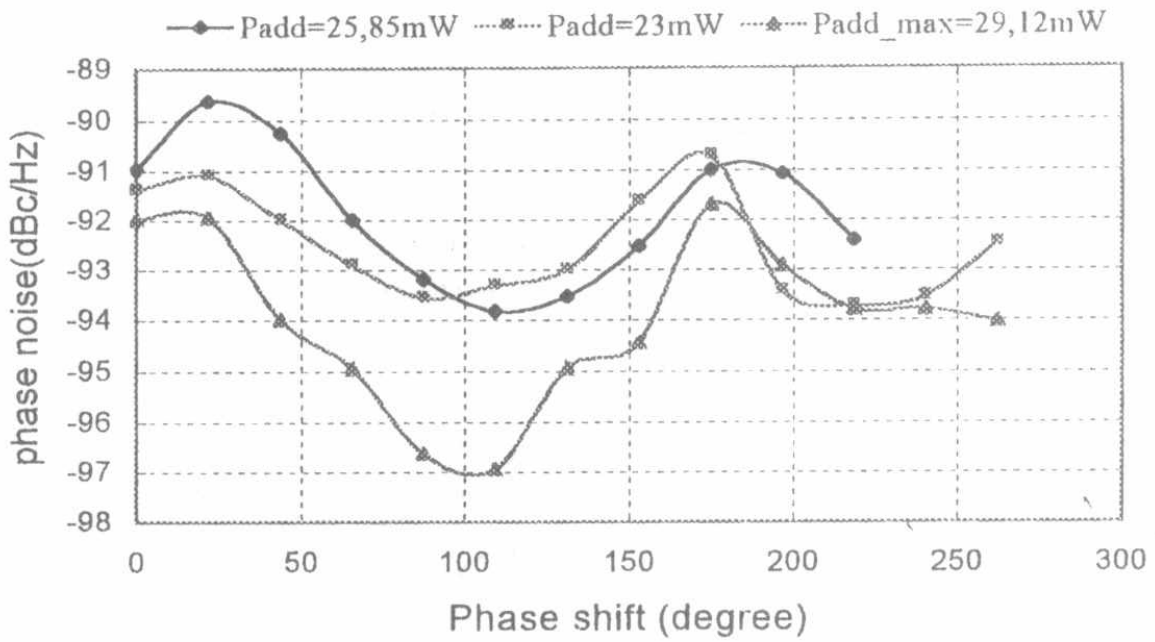

Fig. 8. Transistor adapted in maximum power added

We observe that the curve associated with a maximum power added presents a minimum of phase noise. To compare the influence of the two adaptations (in low level gain and in power added) on noise, we have represented on figure (9) the noise curves fo $r$ the two optimums of power adcied. 


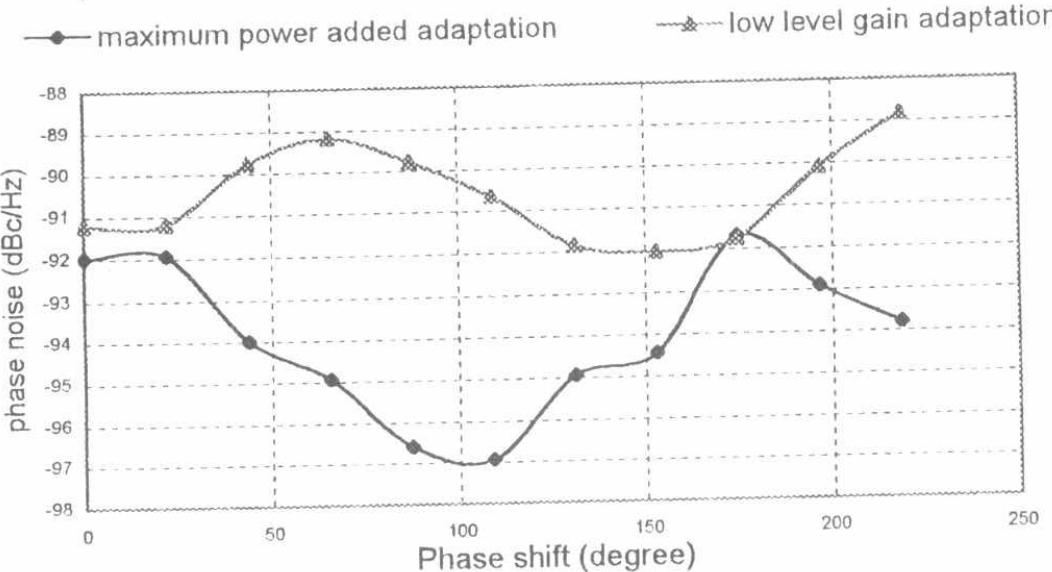

Fig. 9. Two adaptations comparison

We observe that the best result is obtained when the transistor is adapted in maximum power added. We have measured a second PHEMT transistor (MGF4417). The same step has been followed, the transistor is adapted first in low level gain and we measure the oscillator noise versus phase shift, then we adapts the transistor in maximum power added and we measures the phase noise at $10 \mathrm{KHz}$ of the carrier, the results are shown in figure $(10),(11)$ :

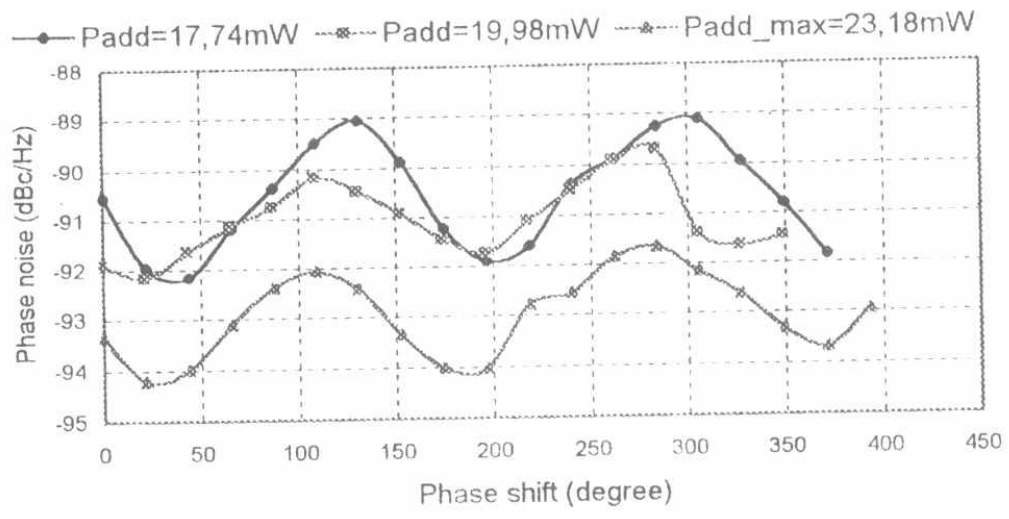

Fig. 10. Transistor adapted in low level gain

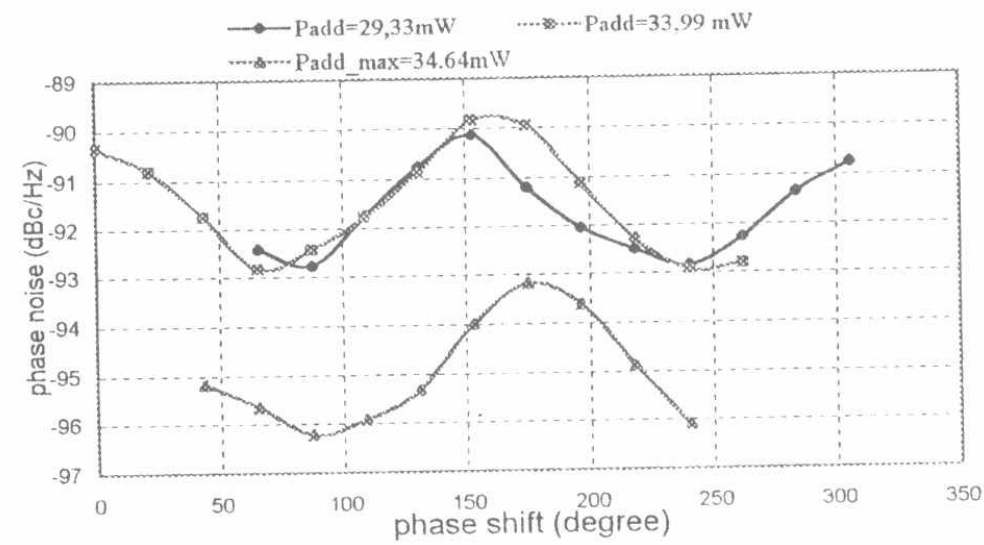

Fig.11. Adaptation in max power added 
We notice for this component also, variations of the phase noise in function of the phase shift brought. The same trends are confirmed, namely that for the two adaptations, the minimum phase noise is cibtained for a maximum power added.

If we compare now levels of phase noise obtained for the adaptation in gain and in power, comparison presented on the figure (12).

We observe that the adaptation presenting a minimum phase noise is the adaptation in power added.

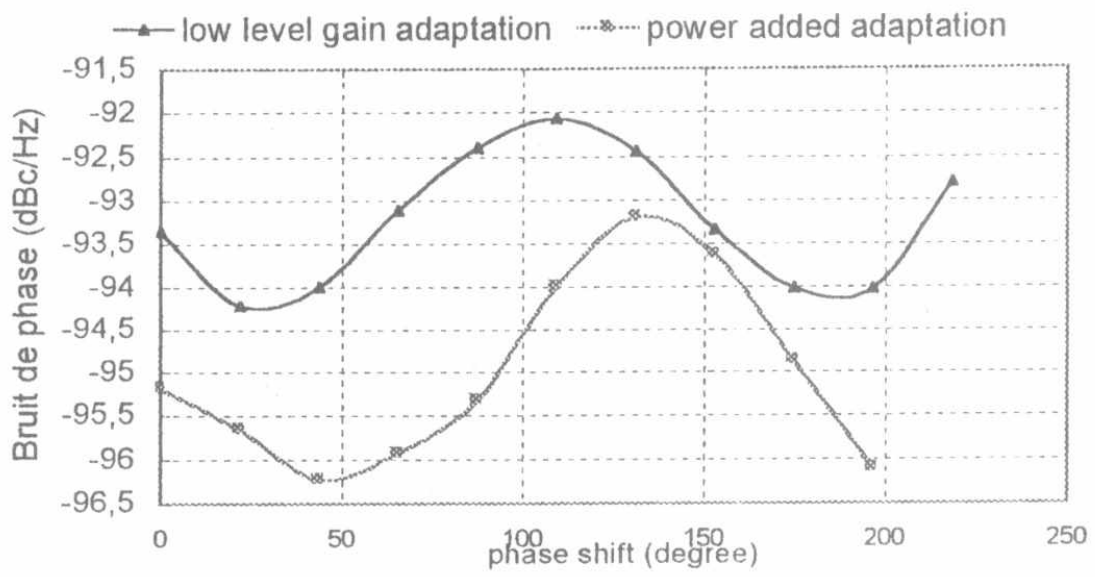

\section{Conclusions}

Fig. 12. Two adaptations comparison

In this communication, we have presented experimental results of a new design method of low phase noise oscillators, method based on the mastery of the nonlinear behavior of the amplifier element, as well as on the choice of a topology of the feedback circuit. We have shown the fact that the transistor functions to maximum power added, allows to dissipate the maximum of power in the cavity, and thus to increase its efficiency, and therefore to reduce the noise of phase.

The choice of an optimal topology of the oscillator, with two phase shifting in the reaction loop associated to an isolator, allows to have the maximal transfer of energy stored between the cavity and the entry of the transistor.

\section{References}

[1] H.Kimouche, "Phase noise measurement and characterization setup of microwaves oscillator for the determination of their optimal working conditions at low phase noise" Thesis of the university of Lirnoges, France, February (1998).

[2] B.Branger, "The optimal circuits conception of minimum phase noise oscillator Application to the realization of an oscillator in $X$ band in the framework of Pharao project ", Thesis of Doctorate of the university of Limoges, January (1998). France

[3] E.L.Holtzman, R.S.Robertson, "Solid state microwave power oscillator design", Artech House, (1992).

[4] R.Soares, "GaAs MESFET circuit design", Artech House, (1988).

[5] T.C.Edwards, "Conception of microwave circuits", Masson, (1984).

[6] R.Goyal, "Monolithic microwave integrated circuit technology and design", Artech House, (1989).

[7] P.Vizmuller, "RF design guide, systems, circuits and equations", Artech House, (1988) 
